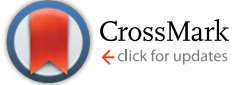

Cite this: RSC Adv., 2014, 4, 64919

Received 26th September 2014 Accepted 18th November 2014

DOI: $10.1039 / c 4 r a 11283 k$

www.rsc.org/advances

\section{Magnetic, X-ray and Mössbauer studies on magnetite/maghemite core-shell nanostructures fabricated through an aqueous route $\dagger$}

\author{
Srividhya J. Iyengar, ${ }^{a}$ Mathew Joy, ${ }^{a}$ Chandan Kumar Ghosh, ${ }^{b}$ Subhrajyoti Dey, ${ }^{c}$ \\ Ravinder K. Kotnala ${ }^{d}$ and Swapankumar Ghosh*a
}

Uniform 6-13 nm sized OD superparamagnetic $\mathrm{Fe}_{3} \mathrm{O}_{4}$ nanocrystals were synthesized by an aqueous 'coprecipitation method' under a $\mathrm{N}_{2}$ atmosphere as a function of temperature to understand the growth kinetics. The crystal phases, surface charge, size, morphology and magnetic characteristics of assynthesized nanocrystals were characterized by XRD, Raman spectroscopy, FTIR, TG-DTA, BET surface area, dynamic light scattering along with zeta potential, HR-TEM, EDAX, vibrating sample magnetometry and Mössbauer spectroscopy. TEM investigation revealed highly crystalline spherical magnetite particles in the $8.2-12.5 \mathrm{~nm}$ size range. The kinetically controlled as-grown nanoparticles were found to possess a preferential (311) orientation of the cubic phase, with a highest magnetic susceptibility of $\sim 57 \mathrm{emu} \mathrm{g}^{-1}$. The Williamson-Hall technique was employed to evaluate the mean crystallite size and microstrain involved in the as-synthesized nanocrystals from the X-ray peak broadening. In addition to FTIR and Raman spectra, Rietveld structural refinement of XRD confirms the magnetite phase with 5-20\% maghemite in the sample. VSM and Mössbauer spectral data allowed us to fit the magnetite/maghemite content to a core-shell model where the shell is $0.2-0.3 \mathrm{~nm}$ thick maghemite over a magnetite core. The activation energy of $<10 \mathrm{~kJ} \mathrm{~mol}{ }^{-1}$ calculated from an Arrhenius plot for the complex process of nucleation and growth by diffusion during synthesis shows the significance of the precipitation temperature in the size controlled fabrication processes of nanocrystals. Brunauer-Emmett-Teller (BET) results reveal a mesoporous structure and a large surface area of $124 \mathrm{~m}^{2} \mathrm{~g}^{-1}$. Magnetic measurement shows that the particles are ferromagnetic at room temperature with zero remanence and zero coercivity. This method produced highly crystalline and dispersed OD magnetite nanocrystals suitable for biological applications in imaging and drug delivery.

\section{Introduction}

Magnetite $\left(\mathrm{Fe}_{3} \mathrm{O}_{4}\right.$, containing $\mathrm{Fe}^{2+}$ and $\mathrm{Fe}^{3+}$ in the $1: 2$ ratio), crystallizes in the inverse cubic spinel structure $F d 3 m$ above the so-called Verwey transition temperature $\sim 120 \mathrm{~K} .^{1}$ The oxygen atoms form the close-packed face-centered-cubic (fcc) lattices with the iron atoms occupying the interstitial positions. ${ }^{2} \mathrm{Fe}_{3} \mathrm{O}_{4}$ nanoparticles exhibit unique and tunable fundamental sizeand shape-dependent novel magnetic, optical, and other unique properties due to quantum confinement effects i.e., the

\footnotetext{
${ }^{a}$ Advanced Clay \& Traditional Ceramics Division, CSIR-Central Glass \& Ceramics Research Institute, Kolkata-700032, India. E-mail: swapankumar.ghosh2@mail.dcu.ie; srividhyaji@gmail.com; Fax: +91 33 24730957; Tel: +91 3323223546

${ }^{b}$ School of Material Science \& Nanotechnology, Jadavpur University, Kolkata-700032, India

'Department of Physics, Jadavpur University, Kolkata-700032, India

${ }^{d}$ Materials Physics and Engineering, CSIR-National Physical Laboratory, New Delhi110012 , India

$\dagger$ Electronic supplementary information (ESI) available. See DOI: $10.1039 / \mathrm{c} 4 \mathrm{ra} 11283 \mathrm{k}$
}

nanometer size effect and have attracted great attention in recent years. The crystal chemistry of $\mathrm{Fe}_{3} \mathrm{O}_{4}$ is of considerable interest to mineralogists and materials scientists because of its extensive applications in magnetic resonance imaging (MRI) for imaging the brain and the central nervous system,,$^{3-5}$ for assessing cardiac function, ${ }^{6}$ as a drug delivery/gene-delivery platform, ${ }^{7}$ ferro-fluids, MICR ink, ${ }^{8}$ cell targeting, ${ }^{9}$ magnetic force based tissue engineering, ${ }^{\mathbf{1 0 , 1 1}}$ magnetically controllable catheters, glucose sensing, ${ }^{12}$ sensing tumor by magnetoimpedance, ${ }^{\mathbf{1 3}}$ and magnetic separation of biological materials. Outstanding magneto-electrical properties have also been reported in strain induced magnetite. ${ }^{\mathbf{1 4 , 1 5}}$ Recent technological advances in smart multifunctional nanobiomagnetic platforms offer exciting opportunities in personalized medicine for more accurate early prognosis, monitoring and treatment of various diseases without jeopardizing healthy tissues by identifying unique biochemical markers of disease before the appearance of symptoms obviates the need. ${ }^{16}$ Nanocrystalline $\mathrm{Fe}_{3} \mathrm{O}_{4}$ often exhibits superparamagnetic behavior. Néel relaxation of superparamagnetic $\mathrm{Fe}_{3} \mathrm{O}_{4}$ is an effective way to heat up the 
nanocrystals and the surrounding tissue by transferring energy from the external magnetic field in therapeutic hyperthermia and, more recently, in the development of theranostic technologies. ${ }^{\mathbf{1 1 7}}$

Large surface area to volume ratio for nanoparticles provides enormous driving force for diffusion, especially at elevated temperatures. ${ }^{18}$ Colombo et al. calculated the activation energy for the reduction of iron oxides (hematite, maghemite and magnetite) at $150^{\circ}$ to $400{ }^{\circ} \mathrm{C}$ under reductive atmosphere. ${ }^{19}$ Brus et al. reported the diffusion controlled aqueous oxidation kinetics of magnetite nanoparticles. ${ }^{20}$ Although magnetite nanocrystals prepared by the precipitation technique have been extensively studied, to the best of our knowledge, seldom efforts have been devoted on its growth kinetics during crystallisation. Magnetite NPs are susceptible to undergo surface oxidation in air to maghemite $\left(\gamma-\mathrm{Fe}_{2} \mathrm{O}_{3}\right)$, and hence the control of magnetic properties at these very small sizes still remains a challenge. Average size estimation by transmission electron microscopy (TEM) is by analyzing rather a limited number of nanoparticles (typically 100-300) compared to $>10^{12}$ NPs investigated by X-ray diffraction (XRD) and vibrating sample magnetometry (VSM). ${ }^{21}$ Debye-Scherrer formula, though widely been used to estimate crystal sizes, underestimates the grain size as it ignores the line broadening due to microstrain in the lattice as a result packing defects/dislocation structures etc. ${ }^{22}$ Application of WilliamsonHall (W-H) method on X-ray profiles not only estimate the crystallite sizes but also isolates the peak broadening due to internal lattice strain. ${ }^{23}$

Over the past couple of decades numerous techniques have been proposed to synthesize nano-sized $\mathrm{Fe}_{3} \mathrm{O}_{4}$ particles, such as sol-gel, ${ }^{24}$ hydrothermal/solvothermal ${ }^{1,25}$ thermal decomposition, ${ }^{26}$ reverse micelles,${ }^{27}$ polyol, ${ }^{28}$ sonolysis,${ }^{29}$ gamma ray irradiation, ${ }^{30}$ microwave plasma synthesis. ${ }^{31}$ Majority of these synthetic methods involves thermal decomposition of organometallic precursors or metal complexes in the presence of surfactants that enables precise control of both size and its distribution. Despite such advantages, lipophilic nature of the nanoparticle (NP) surfaces and cost of reactants presently make these synthetic methods not viable for biomedical applications. ${ }^{21}$ Nevertheless, the aqueous coprecipitation of $\mathrm{Fe}^{2+}$ and $\mathrm{Fe}^{3+}$ salts using an alkali remains the most intensively studied, modified and improved method to find economic and environmental friendly pathways for fabrication of controlled metal oxide nanostructures. Most of the industrial applications of magnetite demand highly magnetic nanoparticles with precise knowledge of size, lattice strain/defects and minimal surface passivation. Crystal dimension of nanomaterials affect key colloid properties such as rheology, film gloss, catalytic activity, chemical sensing etc. ${ }^{32}$ Very often increasing mismatch of magnetic NP size and its saturation magnetization values (and as a result increasing magnetic anisotropy) is reported with decreasing size in magnetite NPs. This is due to the presence of a magnetically dead layer of oxidized 15-30\% amorphous component (with a thickness varying between 0.3 and $1.0 \mathrm{~nm}$ ) over the maghemite shell of magnetite-maghemite core-shell structure. ${ }^{21}$ We show that there are size-dependent changes in the local structure and oxidation state of the oxide shell, the relative fraction of maghemite increasing at the expense of magnetite as the core dimensions decrease. This size/structure correlation has been explained in terms of morphological and structural disorder arguments. ${ }^{33}$

In this communication, we report the estimation of the crystal dimensions of magnetite synthesized through surfactant free aqueous coprecipitation route from the TEM, XRD, and magnetization profiles by Langevin fit. The XRD data were analyzed by Rietveld refinement. The magnetite phase was confirmed from the X-ray, TEM, Raman and Fourier transform infrared (FTIR) spectroscopy. The maghemite content in magnetite was determined from the Mössbauer spectral fitting. The magnetic powder was thoroughly characterized by thermal analyses (TG-DTA), surface area analysis, hydrodynamic size and zeta potential measurements. The activation energy for growth of size tailored magnetite crystals was calculated by employing Arrhenius equation. The crystal dimensions were carefully correlated in terms of maghemite layer content over magnetite core in a core-shell model.

\section{Experimental section}

\section{Materials}

Fe(II) chloride (98\%) and Fe(III) chloride (97\%) were procured from Sigma Aldrich Chemicals. Ammonia solution (25 wt\%) and common solvents $c a$. acetone, ethanol (analytical grade) etc. were purchased from Merck, India. All the syntheses, washings and dilutions were done with Millipore water (Millipore, specific resistivity $18.2 \mathrm{M} \Omega \mathrm{cm}$ (a $25{ }^{\circ} \mathrm{C}$ ). Millipore water for synthetic purposes was purged with XL grade (99.999\%) nitrogen gas for deaeration.

\section{Experimental}

The procedure adopted here is a modification of the method of Shen et $a l^{34}$ To elucidate the formation process of $\mathrm{Fe}_{3} \mathrm{O}_{4}$ nanocrystal in a size tailored manner, $4.2 \mathrm{mmol} \mathrm{FeCl}_{2} \cdot 4 \mathrm{H}_{2} \mathrm{O}$ and $8.4 \mathrm{mmol} \mathrm{FeCl}_{3} \cdot 6 \mathrm{H}_{2} \mathrm{O}$ (such that $\mathrm{Fe}^{3+} / \mathrm{Fe}^{2+}=2$ ) were dissolved in $35 \mathrm{ml}$ deionized water previously deaerated by purging nitrogen gas in a $100 \mathrm{ml}$ three-neck round bottom flask whose central neck was connected to a water-cooled condenser. A thermometer and a glass pipette for $\mathrm{N}_{2}$ gas purge were connected through thermometer pockets to the other two necks. The reaction mixture was heated slowly to $90{ }^{\circ} \mathrm{C}$ over a hot plate magnetic stirrer while continuous stirring. The clear off-yellow color of the suspension turned to muddy orange at $\sim 80{ }^{\circ} \mathrm{C}$ indicating the complete hydrolysis of ferrous and ferric chlorides. After heating the reaction mixture for $\sim 30 \mathrm{~min}$ at $90{ }^{\circ} \mathrm{C}$, $\sim 7 \mathrm{ml} \mathrm{NH}_{3}$ solution was added while vigorous stirring. The orange colored suspension immediately turned black indicating the formation of magnetite crystals. At this point the net $\mathrm{Fe}^{2+}$ concentration was $100 \mathrm{mM}$. The temperature was maintained at $90{ }^{\circ} \mathrm{C}$ for further $30 \mathrm{~min}$ to allow crystal growth. The sequence of magnetite formation from the ferrous and ferric chlorides via hydrated ferrous and ferric oxides during alkali precipitation may be given as $^{35}$ 


$$
\begin{gathered}
\mathrm{FeCl}_{3}+3 \mathrm{NH}_{4} \mathrm{OH} \rightarrow \mathrm{Fe}(\mathrm{OH})_{3}+3 \mathrm{NH}_{4} \mathrm{Cl} \\
\mathrm{FeCl}_{2}+2 \mathrm{NH}_{4} \mathrm{OH} \rightarrow \mathrm{Fe}(\mathrm{OH})_{2}+2 \mathrm{NH}_{4} \mathrm{Cl} \\
\mathrm{Fe}(\mathrm{OH})_{2}+2 \mathrm{Fe}(\mathrm{OH})_{3} \rightarrow \mathrm{Fe}_{3} \mathrm{O}_{4}+4 \mathrm{H}_{2} \mathrm{O}
\end{gathered}
$$

The suspension was cooled naturally to ambient temperature. The resultant $\mathrm{pH}$ of the reaction mixture was $\sim 11$. The entire experiment was carried out carefully under a closed nitrogen atmosphere. The precipitated black solid was collected by magnetic decantation with a help of permanent neodymium iron boron magnet ( $520 \mathrm{G}$ strength), and washed five times with $1: 1$ acetone and methanol mixture. This magnetite is designated as T90. Similarly, magnetites $(\sim 1 \mathrm{~g})$ were produced using the same precursor concentrations at temperatures 33, 45, 60, and $75{ }^{\circ} \mathrm{C}$ under identical conditions and were named as T33, $\mathrm{T} 45$, $\mathrm{T} 60$, and $\mathrm{T} 75$. After the final washing, the precipitates were collected and dried at room temperature in a vacuum oven.

The powder XRD patterns were recorded with Bruker D8 Advanced diffractometer equipped with source $\mathrm{CuK} \alpha_{1}$ radiation $(\lambda=1.5406 \AA)$ with a step size of $0.05^{\circ} 2 \theta$ and a scan speed of $4^{\circ}$ $\min ^{-1}$. The mean crystallite size and microstrain involved in assynthesized nano magneto-crystalline samples were estimated by the Cauchy-Cauchy (CC) approach also known as Williamson-Hall ${ }^{36}$ plot from the linear dependence line-profiling of prominent X-ray diffraction peaks of $\beta_{2 \theta} \cos \theta$ versus $\sin \theta$ as described in eqn (1):

$$
\beta_{2 \theta} \cos \theta=2 \varepsilon \sin \theta+0.9 \lambda / D
$$

where $D$ is the crystal size, $\varepsilon$ is the maximum microdeformation of a lattice, $\beta_{2 \theta}$ is the integral width of the diffraction peaks at angle $\theta$ by the eqn (2):

$$
\beta_{2 \theta}=\frac{I . I}{I_{\mathrm{Max}}}
$$

Strain is estimated from the slope $(2 \varepsilon)$ and the average crystal size $(D)$ from the intercept $(0.9 \lambda / D)$ of the linear regression assuming the particles are spherical. $\mathrm{W}-\mathrm{H}$ analysis is sometimes used as a complementary method to confirm TEM values and to make them more robust from the statistical point of view. We adopted Rietveld powder structure refinement anal$y s i s^{37}$ of X-ray powder diffraction step scan data using the JAVA based program MAUD, ${ }^{38}$ to obtain the structural and microstructural refinement parameters through a least-square method. The experimental profiles are fitted with the most suitable pseudo-Voigt $(\mathrm{pV})$ analytical function ${ }^{37}$ with asymmetry and the background of each pattern is fitted with a fourth order polynomial function.

Standard Harris analysis was performed on X-ray data of magnetite powders ${ }^{39}$ to estimate preferred orientation of specific crystal planes and is expressed as texture coefficient $C\left(h_{i} k_{i} l_{i}\right)$, following eqn (3),

$$
C\left(h_{i} k_{i} l_{i}\right)=\frac{I\left(h_{i} k_{i} l_{i}\right)}{I_{\mathrm{o}}\left(h_{i} k_{i} l_{i}\right)}\left[\frac{1}{n} \sum \frac{I\left(h_{i} k_{i} l_{i}\right)}{I_{\mathrm{o}}\left(h_{i} k_{i} l_{i}\right)}\right]^{-1}
$$

where, $I\left(h_{i} k_{i} l_{i}\right)$ is the diffraction intensity of the $\left(h_{i} k_{i} l_{i}\right)$ plane of the particular sample under investigation, $I_{\mathrm{o}}\left(h_{i} k_{i} l_{i}\right)$ is the intensity of the $\left(h_{i} k_{i} l_{i}\right)$ plane from the standard JCPDS powder diffraction pattern for the corresponding peak $i$, and $n$ is the number of reflections taken in to account. FTIR spectra on magnetite samples were taken at room temperature on a PerkinElmer Spectrum 100 spectrophotometer in the $400-4000 \mathrm{~cm}^{-1}$ range with average of 50 scans. The powder specimens were pressed into small discs using spectroscopically pure $\mathrm{KBr}$ (Sigma-Aldrich, $\geq 99 \%$ ) matrix with sample to $\mathrm{KBr}$ ratio $\sim 1: 100$ to evaluate the structural aspects of magnetite. The Raman spectrometer is equipped with an optical microscope (Olympus Confocal Raman Optics micrometer), a thermoelectrically cooled $\left(-60{ }^{\circ} \mathrm{C}\right)$ charge-coupled device (CCD) detector and a CCD camera (resolution $1340 \times 1100)$ that can provide a good laser beam. The Raman spectra were collected by $514.5 \mathrm{~nm}$ radiation from an argon ion laser (Stellar Pro, $50 \mathrm{~mW}$ ) on Princeton Instruments Acton SP2500. Samples were compacted into a $1 \mathrm{~mm}$ cavity held on an anodized aluminum plate. The laser beam was focused on the sample by a $\times 50$ lens to a spot size of $c a$. 5-6 $\mu \mathrm{m}$. Coaxial backscatter geometry was employed for signal collection with spectral resolution of $\sim 1 \mathrm{~cm}^{-1}$. The Raman shifts were calibrated using the $520 \mathrm{rcm}^{-1}$ line of a silicon wafer. The spectra were collected using $2.5 \mathrm{~mW}$ laser power over the range $1000-200 \mathrm{rcm}^{-1}$ and accumulation over 10 scans, each with an exposure time of $10 \mathrm{~s}$ to reduce noise further. Crystal dimensions can also be estimated from the magnetization curves based on the theory of superparamagnetism as proposed by Bean and co-workers. ${ }^{40-42}$ To fit the magnetization curves, we assume that individual grains are single crystals without mutual interaction and each particle has an inner single-domain core with the spontaneous magnetization. The magnetization of $N$ number of ideal non-interaction superparamagnetic nanoparticles, each with identical magnetic moment $\mu$, at constant temperature $T$ in magnetic field $H$ is given by Langevin function eqn (4),

$$
M(H)=N \mu\left\{\left(\frac{\mathrm{e}^{2\left(\frac{\mu H}{k_{\mathrm{B}} T}\right)}+1}{\mathrm{e}^{2\left(\frac{\mu H}{k_{\mathrm{B}} T}\right)}-1}\right)-\frac{k_{\mathrm{B}} T}{\mu H}\right\}
$$

where $k_{\mathrm{B}}$ and $N \mu$ are the Boltzmann constant and saturation magnetization $\left(M_{\mathrm{s}}\right)$ of the synthesized sample.

The magnetization curves were fitted using a nonlinear-least squares routine to obtain two parameters: the log-mean single particle moment, $\mu$, and $M_{\mathrm{s}}$. The size, called "magnetic size", is significantly smaller than the physical size obtained from TEM. Magnetic measurements of the powder sample were made using VSM (Lakeshore 7305, US) at $298 \mathrm{~K}$ and Physical Property Measurement System (PPMS, Cryogen, UK). ${ }^{57} \mathrm{Fe}$ Mössbauer spectra of the two selected specimens T33 and T90 were recorded at room temperature by means of a standard constant acceleration transmission mode with a $\sim 50 \mathrm{mCi}{ }^{57} \mathrm{Co}$ diffused in rhodium matrix using a $\alpha$-Fe foil for calibration. The experimental profiles were fitted to Lorentzian functions by leastsquare method with RECOIL software package. The average size and the morphologies of magnetite nanoparticles and its 
crystal structure were ascertained by the high resolution transmission electron microscopy (HR-TEM) using a FEI Tecnai 30 $\mathrm{G}^{2} \mathrm{~S}$-Twin HR-TEM operated at $300 \mathrm{kV}$ equipped with a Gatan CCD camera. The chemical composition was determined on several crystal grains by using an EDAX spectrometer equipped with high-angle annular dark-field detector with beam scanning capability (Fischione Instruments, Inc., USA) with TIA analysis software. The standard deviation of size was calculated using the formula eqn (5),

$$
\sigma=\sqrt{\frac{1}{N} \sum_{i=1}^{N}\left(x_{i}-\bar{x}\right)^{2}}
$$

where $N$ is sampling number, $x_{i}$ is random variable, $x^{-}$is mean size and the size distribution is calculated using probability density eqn (6), based on a log-normal function

$$
p(D)=\frac{1}{D \sigma_{1} \sqrt{2 \pi}} \exp \left\{-\frac{\left[\ln \left(D / D_{0}\right)\right]^{2}}{2 \sigma_{1}{ }^{2}}\right\}
$$

where $\sigma$ is standard deviation, $D$ is size, and $D_{0}$ is the mean size.

The specific surface area and pore size distribution of the magnetite powders were determined from the $\mathrm{N}_{2}$ adsorption data following BET technique at $77 \mathrm{~K}$ using a surface area analyser (Quantachrome Instruments version 10.01). Surface area analyses were conducted on powder samples after degassing them at $200{ }^{\circ} \mathrm{C}$ for $3 \mathrm{~h}$. The thermogravimetric (TG) analysis was used to investigate the thermal reactions of the synthesized nanocrystals using a Simultaneous Thermal Analyzer (STA-6000, Perkin-Elmer, The Netherlands) under ultrapure nitrogen purge. The thermograms were collected with a ramp of $10{ }^{\circ} \mathrm{C} \mathrm{min}^{-1}$ in the temperature range $50-1000{ }^{\circ} \mathrm{C}$.

\section{Results and discussion}

Magnetite nanoparticles were synthesized by the economic, non-toxic aqueous coprecipitation method at different reaction temperatures in the range $33^{\circ}$ to $90{ }^{\circ} \mathrm{C}$. The synthesized iron oxide powders (T33 to T90) were typically black in color indicating the presence of magnetite as the dominant phase, although ancillary $\gamma-\mathrm{Fe}_{2} \mathrm{O}_{3}$ may not be completely excluded. The bright field HR-TEM images for selected samples T90, T75, T60 and T33 are shown in Fig. 1 (additional images are provided in Fig. S1 in the ESI $\dagger$ ). TEM images show spherical, crystalline and well resolved particles with narrow size distribution in 6-13 nm range. Apparently the particles do not interact with each other at room temperature which will be further investigated by the magnetization measurements in a later section (Fig. 3). The TEM results shown in Fig. 1 reveal that the precipitation temperature increases the average particle size of T33 from 7.76 $\pm 1.94 \mathrm{~nm}$ to $10.84 \pm 1.97 \mathrm{~nm}$ in $\mathrm{T} 60$, while $75{ }^{\circ} \mathrm{C}$ reaction temperature further increases the size to $12.32 \pm 1.63 \mathrm{~nm}$.

On the other hand, the magnetite synthesized at $90{ }^{\circ} \mathrm{C}$ doesn't improve the equilibrium size much but most particles fall in relatively wider $12.42 \pm 1.87 \mathrm{~nm}$ size range (Fig. 1A). The particle size and number based size distribution of
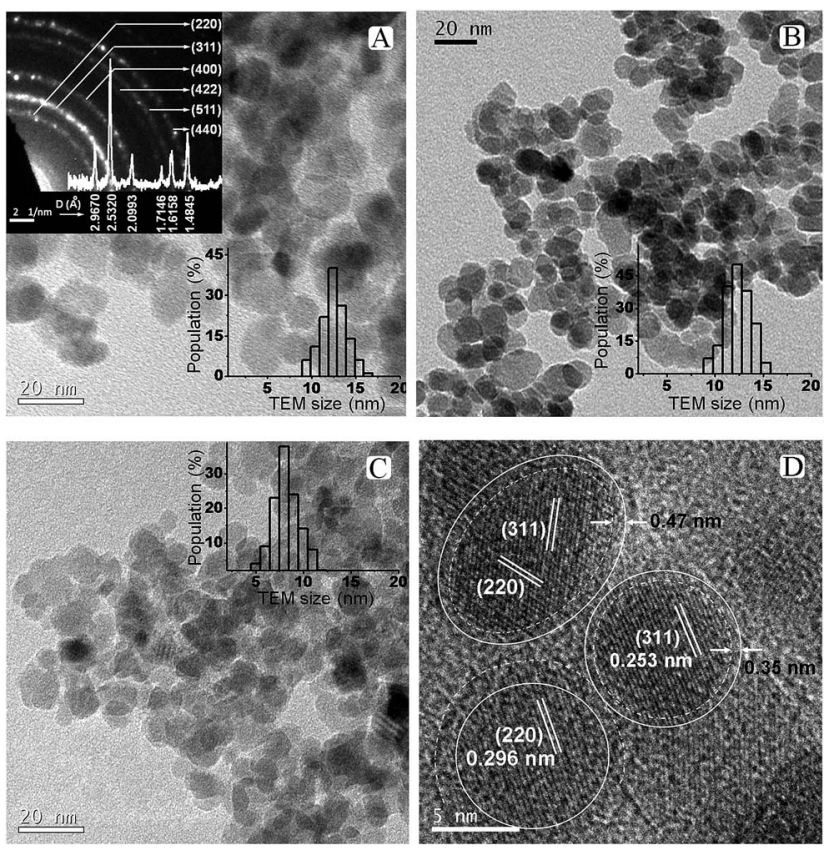

Fig. 1 Bright-field TEM images of as synthesized magnetite nanoparticles (A) T90 with its SAED pattern (inset), (B) T75, (C) T33, and (D) HR-TEM of T33 indicating clear crystalline core surrounded by amorphous shell (marked with white line). The size distribution histograms of the corresponding nanocrystals for all the images are presented as inset to each image.

nanoparticles are calculated by probability density function (eqn 6) from the inspection of multiple TEM images on 100-200 particles. These results suggest that the size of the resultant $\mathrm{Fe}_{3} \mathrm{O}_{4}$ nanocrystals is strongly dependent on the precipitation temperature. Careful examination of the high resolution images indicates that all the nanoparticles are single crystals and fringes corresponding to predominant (311) and (220) planes could be identified. Selected area diffraction (SAED) patterns of T90 (inset of Fig. 1A) shows clear Debye-Scherrer rings corresponding to (220), (311), (400), (422), (511) and (440) planes with corresponding interplanar spacings 0.2967, 0.2532, 0.2099, $0.1715,0.1616$ and $0.1485 \mathrm{~nm}$ respectively (JCPDS card no. 190629). The careful observation of HR-TEM images clearly reveals crytallographically different core-shell morphology in nanocrystals of T33 with amorphous shell of thickness $\sim 0.3-0.5 \mathrm{~nm}$. The atomic ratio of Fe:O of the nanoparticles measured by EDAX (Fig. S1 in ESI†) is $3: 4$. Within the limits of sensitivity $(\leq 3 \%)$, these EDAX data also suggest that the nanoparticles have no detectable impurities: the signal from $\mathrm{C}$ and $\mathrm{Cu}$ was due to the carbon film over the copper TEM grid.

The X-ray diffraction patterns of $\mathrm{Fe}_{3} \mathrm{O}_{4}$ synthesized at different temperatures and Rietveld analysis on the XRD pattern of T33 are shown in Fig. 2. All Bragg's planes could be indexed to face centered cubic inverse spinel magnetite of $F d 3 m$, \#227 space group with lattice constant $a$ in the range $0.8340-0.8366$ $\mathrm{nm}$ whereas the same for bulk magnetite is $0.8396 \mathrm{~nm}$ (JCPDS card no. 19-0629). 

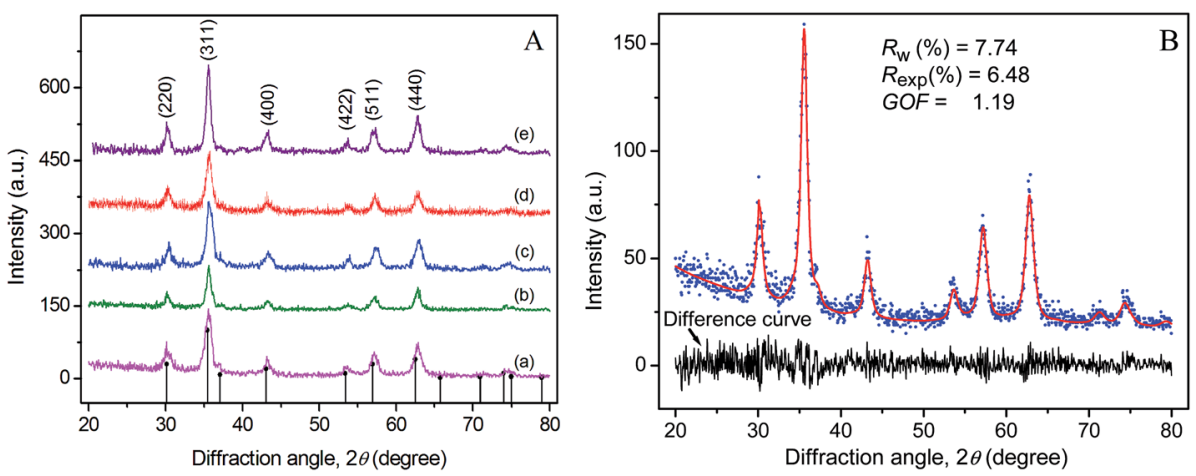

Fig. 2 (A) X-ray diffraction patterns of magnetites (a) T33, (b) T45, (c) T60, (d) T75, and (e) T90. The vertical drop lines in (A) are the theoretical Bragg positions for inverse spinel magnetite phase following JCPDS Card no. 19-0629. (B) is a typical Rietveld fit (solid line) for T33 over the corresponding raw XRD data (dots). The noisy layer in (B) is the difference curve between the XRD profile and the Rietveld simulation.

Broad diffraction peaks (Fig. 2A) indicate the nanocrystalline nature of the particles. When the reaction temperature was increased in steps from 33 to $90{ }^{\circ} \mathrm{C}$, a slight progressive narrowing of the X-ray diffraction peaks occurred as a result of crystal growth and reduction of microstrain in the lattice originating from defects. The match of the X-ray pattern with its Rietveld fit and the resultant almost spike less difference pattern indicates the quality of fit in magnetite-maghemite mixed phases in the synthesized sample. The Rietveld analyses of X-ray patterns of T33 and T90 (Fig. S4 in ESI $\dagger$ ) allowed us to estimate the relative concentrations of magnetite phase as 89.2 and $87.3 \%$ respectively which is compatible with a core-shell model where the shell is $0.2-0.3 \mathrm{~nm}$ thick in $D_{\text {TEM }}=8.2$ and $12.5 \mathrm{~nm}$ particles in T33 and T90 respectively. The core-shell structure was also observed from HR-TEM images (Fig. 1). The fit parameters are consistent with reported data in the literature. ${ }^{43}$ Fig. 3 shows the field dependent magnetic properties of the synthesized $\mathrm{Fe}_{3} \mathrm{O}_{4}$ samples (T33 and T90), measured by VSM at room temperature as well as PPMS at different temperatures with higher field resolution. The hysteresis loops apparently pass through the origin which indicates zero coercivity and zero remanence (Fig. 3A), signifying that the samples are in the superparamagnetic state with unstable magnetization at this temperature ${ }^{44}$ and the samples possess higher magnetization at $250 \mathrm{~K}$ due to reduced thermal energy (Fig. 3B).

In contrast to saturated magnetization $\left(M_{\mathrm{s}}\right)$ of ferromagnetic $\mathrm{Fe}_{3} \mathrm{O}_{4}$ nanoparticles, measured by Tian et al., ${ }^{45}$ we observed unsaturated magnetic behavior in our synthesized nanoparticles that confirms the superparamagnetic nature. On careful examination of the high resolution magnetization data (Fig. 3B) it is observed that both T33 and T90 display coercivities to the extent of mere 21 and 16 Oe respectively. Appearance of the coercivity may be attributed to the long range magnetic dipolar interactions. The magnetization profiles as a function of $H / T$ (inset of Fig. 3B) are very much close to each other suggesting non-interactive nature of the particles. However, small deviation between the curves in the high field region and the presence of coercive field indicate that the mutual interaction is not absolutely zero i.e., a weak interaction exists among them. ${ }^{\mathbf{4 6}}$ Due to the asymptotic increase of magnetization for high fields (see Fig. 3), the saturation magnetization value can be obtained from the fitting of the $M v s$. $1 / H$ curves, extrapolating the magnetization value to $1 / H=0 .{ }^{47}$ According to inset of Fig. 3A, the observed magnetization for T90 and T33 are 48.44 and 56.11 emu $\mathrm{g}^{-1}$ respectively. The curves were fitted with Langevin function in order to get $M_{\mathrm{s}}$ of samples and the obtained fitting parameters are summarized in Table 1 . It is noteworthy to
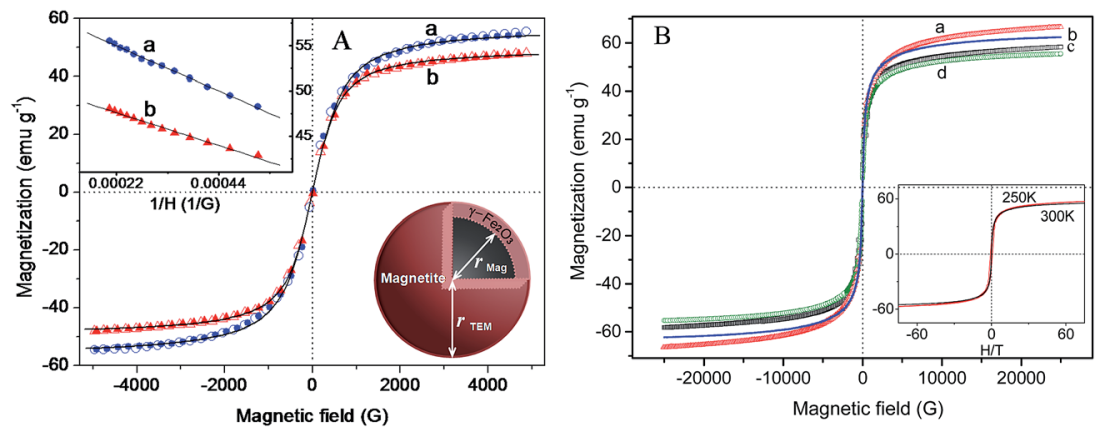

Fig. 3 Magnetization versus applied magnetic field data (A) with low field resolution for selected magnetite (a) T33 and (b) T90 specimens and (B) higher field resolution from PPMS at (a) T33 at $250 \mathrm{~K}$, (b) T33 at $300 \mathrm{~K}$, (c) T90 at $250 \mathrm{~K}$ and (d) T90 at $300 \mathrm{~K}$ and its inset represents the magnetization data as a function of $H / T$ for T90. Typical Langevin fits are represented by solid black lines in (A). 
mention that the $M_{\mathrm{s}}$ of our synthesized samples is greater than that of $\mathrm{Fe}_{3} \mathrm{O}_{4}$ nanosheets of thickness $\sim 10 \mathrm{~nm}$ and nanorods of length $>100 \mathrm{~nm}$ and diameter of $\sim 10 \mathrm{~nm},{ }^{48}$ but is quite less than bulk $\mathrm{Fe}_{3} \mathrm{O}_{4}$ (92 emu $\left.\mathrm{g}^{-1}\right)^{49}$ and the reduction of $M_{\mathrm{S}}$ may be attributed to the disordered surface spin or spin canting behavior at the surface of the nanoparticles and change in degree of inversion.

It is worthy to mention that $M_{\mathrm{S}}$ of T33 is higher than that of T90 i.e., bigger particle has lower value of $M_{\mathrm{s}}$. The magnetic sizes of $\mathrm{T} 90\left(D_{\mathrm{mag}}=10.83 \mathrm{~nm}\right)$ from our computations are smaller than the physical size measured by TEM $\left(D_{\text {TEM }}=12.42\right.$ $\mathrm{nm})$. It is reasonable to assume that the difference may be attributed to the presence of magnetically inactive outer layer that is responsible for suppression of magnetization in our synthesized samples. ${ }^{47,50}$ As it is well known that $\mathrm{Fe}_{3} \mathrm{O}_{4}$ having inverse spinel structure in bulk possesses mixed spinel structure in nano-phase and the degree of inversion decreases with increase in particle size. Since T90 has larger size than T33, the reduction of magnetization in case of T90 may be ascribed to the reduction of the degree of inversion. Interestingly, it has been observed that $D_{\text {TEM }}$ for T33 $(8.2 \mathrm{~nm})$ is smaller than $D_{\text {mag }}$ $(9.86 \mathrm{~nm})$ i.e., magnetic size is overestimated by approximately $10 \%$ in comparison to TEM size. Such overestimation was previously observed by few researchers and can be explained on the basis of either perturbation in the Langevin function caused by interparticle interactions that was ignored during fitting ${ }^{51}$ or the consideration of single size distribution of the particles. In order to get a better information, the magnetic sizes $\left(D_{\text {mag }}\right)$ were also calculated by using eqn (7), ${ }^{52,53}$

$$
D_{\mathrm{mag}}=\left(\frac{18 k T}{\pi} \frac{\chi}{\rho M_{\mathrm{s}}^{2}}\right)^{\frac{1}{3}}
$$

where $\chi$ and $\rho$ represent susceptibility $\left(\frac{\mathrm{d} M}{\mathrm{~d} H}\right)_{H \rightarrow 0}$ and density of the material respectively. Eqn (7) gives $D_{\text {mag }}$ as $6.6 \mathrm{~nm}$ for T33 and remove the discrepancy that emerged from the Langevin curve analysis.

${ }^{57}$ Fe Mössbauer spectra of T33 and T90 samples shown in Fig. 4 consist of two sextets and a single doublet pattern depending on the size and crystallinity of the particles. The outer sextet of smaller area corresponds to $\mathrm{Fe}^{3+}$ in tetrahedral (A) sites while the inner sextet with larger area corresponds to $\mathrm{Fe}^{3+}$ and $\mathrm{Fe}^{2+}$ in octahedral (B) sites. However, the weak doublet peak signifies the presence of some particles lying below the critical size for superparamagnetic relaxation at the measurement time-scale. The average hyperfine magnetic field (HMF) decreased in the magnetic powder specimens from T33 synthesized at $33{ }^{\circ} \mathrm{C}$ to T90 prepared at $90{ }^{\circ} \mathrm{C}$ (Table 2). This drop may be attributed to the lower net magnetic moment per unit formula for T90 as determined by the $\mathrm{Fe}^{3+}$ ion concentration at octahedral site in comparatively larger particles. The relative area ratio of two $\mathrm{Fe}^{3+}$ sextets for both the samples reveals that the concentration of $\mathrm{Fe}^{3+}$ ions at the octahedral (B) site is lower than that at the tetrahedral (A) site.

The isomer shift $(\delta)$ values corresponding to tetrahedral $\left(\delta_{\mathrm{A}}\right)$ and octahedral $\left(\delta_{\mathrm{B}}\right)$ sites of the magnetite specimens (Table 2) illustrate that the s-electron density at the Mössbauer active nuclear site is significantly affected by the rise in precipitation temperature. Nevertheless, the value of the isomer shift of $\mathrm{Fe}^{3+}$ in the A site is higher than that generally reported for the micrometric bulk magnetite, ${ }^{\mathbf{1}}$ indicating a possible charge transfers in the A site also.

Negative quadrupole shift (QS) for both the samples may be attributed to the oblate charge distribution of Fe. The ferric character of the Fe ion is also manifested by the magnitude of the magnetic hyperfine fields and is proportional to the spin of the ferric ion. ${ }^{45}$ The sextet area leading to a $\mathrm{Fe}_{\text {tetra }}{ }^{3+} / \mathrm{Fe}_{\text {octa }}{ }^{3+, 2+}$ ratio of T33 and T90 is found to be $0.80(42.5 / 52.8)$ and 0.85 respectively, in contrast to the theoretical ratio 0.50 . Such intensity ratios, which exceeds 0.50 , are called super stoichiometry in oxygen or cationic vacancies. ${ }^{1}$ In contrast to relative intensity ratio $1: 2$ corresponding to Fe between $\mathrm{A}$ and $\mathrm{B}$ sites in bulk inverse spinel magnetite, $\left(\mathrm{Fe}^{3+}\right)_{\mathrm{A}}\left[\mathrm{Fe}^{2+} \mathrm{Fe}^{3+}\right]_{\mathrm{B}} \mathrm{O}_{4}$, we observed the global composition of T33 and T90 as,

$$
\begin{gathered}
\text { T33: }\left(\mathrm{Fe}^{3+}\right)_{\mathrm{A}}\left[\mathrm{Fe}_{0.814}{ }^{2+} \mathrm{Fe}_{1.124}{ }^{3+} \Phi_{0.062}\right]_{\mathrm{B}} \mathrm{O}_{4} \\
\text { T90: }\left(\mathrm{Fe}^{3+}\right)_{\mathrm{A}}\left[\mathrm{Fe}_{0.79}{ }^{2+} \mathrm{Fe}_{1.14}{ }^{3+} \Phi_{0.07}\right]_{\mathrm{B}} \mathrm{O}_{4}
\end{gathered}
$$

where $\Phi$ represents the cation vacancy at the B-site. The stoichiometry implies that the $\mathrm{Fe}_{3} \mathrm{O}_{4}$ content in T33 sample was $81.4 \%$ and the rest being $\mathrm{Fe}_{2} \mathrm{O}_{3}$. T90 contains slightly lower amount of magnetite at $79 \%$. The relatively larger fraction of oxidized $\mathrm{Fe}^{3+}$ (maghemite) in T90 may be attributed to the dominating effect of higher precipitation temperature though T33 has a larger fraction of surface atoms. The $\Phi$-value clearly indicates the formation of non-stoichiometric magnetite with some $\mathrm{Fe}^{2+}$ deficient lattice sites. If we assume the overall shape of the magnetic nanocrystals are spherical and the oxidized $\gamma$ $\mathrm{Fe}_{2} \mathrm{O}_{3}$ phase exists as shell of uniform thickness over magnetite in a core-shell model, one can easily compute the $18.6 \%$ maghemite in a $8.2 \mathrm{~nm}$ crystal to $\sim 0.27 \mathrm{~nm}$ thick shell as shown in Fig. 4. Similarly, 79\% magnetite content in $12.4 \mathrm{~nm}$ T90 is compatible with $11.6 \mathrm{~nm}$ core/0.47 nm shell (Fig. 4B). The thicknesses of maghemite shells obtained from magnetization studies are close match to the same observed from TEM

\begin{tabular}{|c|c|c|c|c|c|c|}
\hline Sample & $\operatorname{Exp} M_{\mathrm{s}}\left(\mathrm{emu} \mathrm{g}^{-1}\right)$ & $\mathrm{Cal} M_{\mathrm{s}}\left(\mathrm{emu} \mathrm{g}^{-1}\right)$ & $\mu\left(\mathrm{emu} \mathrm{NP}^{-1}\right)$ & $R^{2}$ & Standard error & $D_{\text {mag }}(\mathrm{nm})$ \\
\hline $\mathrm{T} 33$ & 56.11 & 57.22 & $1.48869 \times 10^{-16}$ & 0.9995 & \pm 1.04 & 9.86 \\
\hline T90 & 48.44 & 49.86 & $1.71487 \times 10^{-16}$ & 0.9998 & \pm 0.54 & 10.83 \\
\hline
\end{tabular}
micrography. The formation process of maghemite shell over

Table 1 The detailed analysis from the Langevin fit of the magnetite samples 

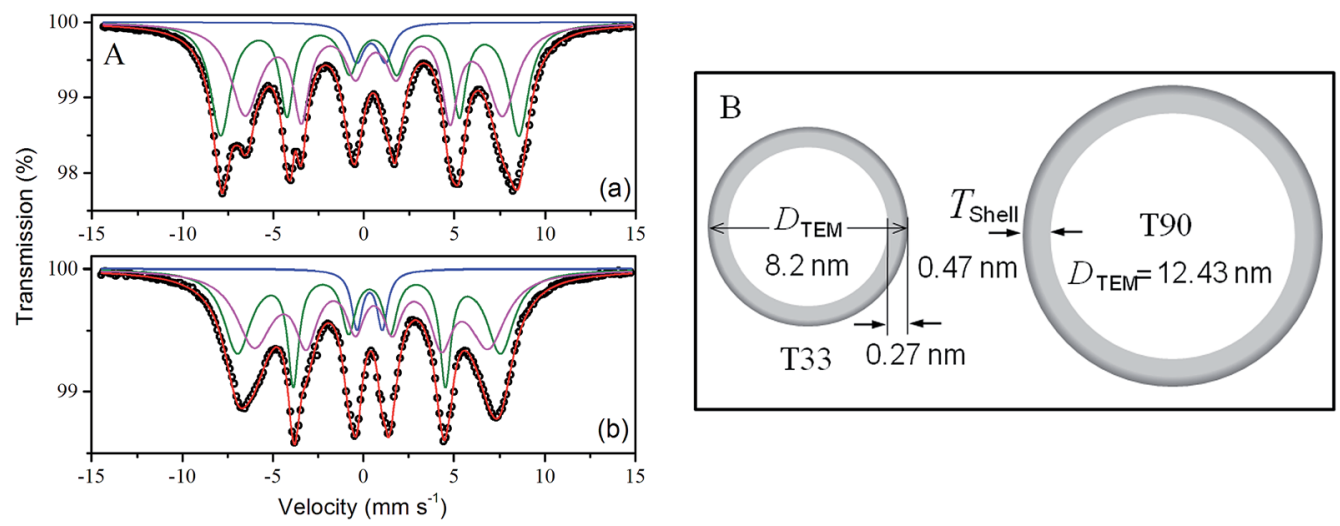

Fig. 4 (A) ${ }^{57}$ Fe transmission Mössbauer spectra of (a) T33 and (b) T90 recorded at room temperature. Symbols represent the experimental data and the continuous lines correspond to the simulated data and (B) schematic representation of core-shell frame-works in T33 and T90.

magnetite core begins by dissociative oxygenation of $\mathrm{Fe}^{2+}$ cations at the surface. This phenomenon generates surface $\mathrm{Fe}^{3+}$ cations along with cation vacancies. The $\mathrm{Fe}^{2+}$ ions diffuse out from the inner oxide core to the surface to attain the $\mathrm{Fe}^{3+}$ state. During this process, the outer oxide layer thickens and a radial compositional gradient is established producing a core-shell structure, with maghemite being more abundant in proximity to the surface of the particles forming shell. In order to observe superparamagnetism, as shown in Fig. 3, the time-scale of the measurement $t_{\mathrm{m}}$ should exceed the superparamagnetic relaxation time $(\tau)$, which is usually in the order of $10^{-9}$ to $10^{-10} \mathrm{s.}^{\mathbf{1 0}}$ The critical size $D_{\mathrm{c}}$ of the particles to be superparamagnetic may be calculated empirically from the equation $D_{\mathrm{c}}=\left(\ln t_{\mathrm{m}} / \tau\right)^{1 / 3}$. In the measurement of magnetization, the observation time $t_{\mathrm{m}} \sim 1$ $\mathrm{s}$, and hence the superparamagnetic relaxation can be observed in particles with of size $10^{1}$ to $10^{2} \mathrm{~nm}$. In a Mössbauer study the measurement time is much shorter $\left(t_{\mathrm{m}} \sim 10^{-8} \mathrm{~s}\right)$, and therefore manifestation of superparamagnetism is expected in particles of much finer sizes. ${ }^{54}$

The grain sizes obtained from the $\mathrm{W}-\mathrm{H}$ plots showed almost linear increase with increasing precipitation temperature indicating crystal growth during precipitation (Fig. S2 in ESI $\dagger$ ). A steady increase in the lattice strain was observed (in the range $4.0-7.2 \times 10^{-3}$ ) with decrease in the particle size (Fig. S2 $\dagger$ ) ${ }^{55}$ It is interesting to note that the estimated $\left(D_{\mathrm{XRD}}\right)$ from the $\mathrm{W}-\mathrm{H}$ technique for all the nanocrystals are within $D_{\mathrm{TEM}}-2.5 \mathrm{~nm}$ as the shell of amorphous layer has not contributed to the X-ray diffraction. The deviation is more in the crystals synthesized at higher temperatures. Atoms at the surface and edges are under-coordinated with the presence of broken bonds. As the crystal dimension decreases, the surface area-to-volume ratio increases resulting in a increased broken bond density at the surface causing the remaining bonds to contract spontaneously with an associated increase in bond strength, which in turn produces localized strain. ${ }^{56} \mathrm{~A}$ decrease in the lattice parameter to $8.3596 \AA$ was observed when the particle size increased from 7.3 to $8.4 \mathrm{~nm}$ as a result of possible higher surface oxidation of magnetite crystals to $\gamma-\mathrm{Fe}_{2} \mathrm{O}_{3}$. The cell constant decreased slightly further to $\sim 8.3544 \AA$ on increasing size to $9.7 \mathrm{~nm}$ and remained almost constant thereafter. W-H analysis of X-ray data confirmed that the lattice parameter and crystal lattice strains in magnetite nanoparticles are primarily dependant on the crystal sizes. The preferential orientation of the crystallites along different crystal planes $(h k l)$, texture coefficients, $C(h k l)$ in the $\mathrm{Fe}_{3} \mathrm{O}_{4}$ nanocrystals (Fig. $\mathrm{S} 3 \dagger$ ) shows preferentially grown $\{110\}$ planes to texture coefficient value of 1.323 in T45 because of the growth confinement of (311), (440) and (511) crystal facets and reduced slightly further to 1.288 in T90 (synthesized at $90{ }^{\circ} \mathrm{C}$ ). $\mathrm{Fe}_{3} \mathrm{O}_{4}$ with predominant active (220) planes (T45 to T90) is of great potential in catalytic applications. A $C(h k l)$ value of

Table 2 Values of room temperature ${ }^{57} \mathrm{Fe}$ Mössbauer parameters by Lorentzian site analysis using Recoil program

\begin{tabular}{|c|c|c|c|c|c|c|}
\hline Sample & Site & $\begin{array}{l}\text { Isomer shift, } \delta^{a} \\
\left(\mathrm{~mm} \mathrm{~s}^{-1}\right)\end{array}$ & $\begin{array}{l}\text { Quadrupole splitting, } \Delta E_{\mathrm{Q}}{ }^{a} \\
\left(\mathrm{~mm} \mathrm{~s}^{-1}\right)\end{array}$ & $\begin{array}{l}\text { Average hyper fine field, } B_{\mathrm{hf}}{ }^{b} \\
\text { (Tesla) }\end{array}$ & $\begin{array}{l}\text { Width }^{a} \\
\left(\mathrm{~mm} \mathrm{~s}^{-1}\right)\end{array}$ & $\begin{array}{l}\text { Area fraction }{ }^{c} \\
(\%)\end{array}$ \\
\hline \multirow[t]{2}{*}{ T33 } & Doublet & 0.40 & 1.60 & - & 0.45 & 4.7 \\
\hline & Sextet $[\mathrm{B}]$ & 0.60 & -0.06 & 44.0 & 0.65 & 52.8 \\
\hline \multirow[t]{2}{*}{ Т90 } & Doublet & 0.35 & 1.40 & - & 0.35 & 6.2 \\
\hline & Sextet (A) & 0.32 & -0.02 & 45.1 & 0.45 & 43.1 \\
\hline
\end{tabular}

${ }^{a}$ Standard deviation equal to $\pm 0.03 .{ }^{b}$ Standard deviation equal to $\pm 0.4 .{ }^{c}$ Standard deviation equal to \pm 0.2 . 
1 indicates a particle with randomly oriented crystallites, while a larger value indicates an abundance of crystallites oriented to that $(h k l)$ plane. ${ }^{57}$ The high-index planes usually have higher surface energy. The energy for different crystal planes is in the order $\gamma(111)<\gamma(100)<\gamma(110)<\gamma(220)$ for the face-centeredcubic magnetite phase. ${ }^{58}$

The Arrhenius plot for the samples prepared at temperatures in the range $33-90{ }^{\circ} \mathrm{C}$ (Fig. S4 $\dagger$ ) gave the activation energy from the gradient of the linear regression as $6.15 \mathrm{~kJ} \mathrm{Mol}^{-1}$ under the condition of homogeneous growth of nanocrystallites. This energy is responsible for initiating the complex process of nucleation and growth by diffusion as well as secondary growth by Ostwald ripening. In the case of ammonia precipitated magnetite crystals, the activation energy is relatively small as the nanocrystals have large surface area and its poor crystallinity. The growth process involves a dissolution-crystallisation mechanism allowing a decrease of the free enthalpy of the system by reduction of the surface area. ${ }^{42}$

FTIR spectra on selected magnetite specimens conducted in air are shown in Fig. 5. FTIR spectra also confirm the presence of fcc magnetite in the materials as was previously confirmed from the TEM and XRD data (Fig. 1 and 2). The IR absorption bands in the $630-550 \mathrm{~cm}^{-1}$ range is attributed to the vibrations of $\mathrm{Fe}-\mathrm{O}$ bonds in tetrahedral and octahedral sites ${ }^{59}$ and must have been resulted from the split of the $\nu_{1}$ band at $\sim 570 \mathrm{~cm}^{-1}{ }^{60}$ The band at $\sim 440 \mathrm{~cm}^{-1}$ in T90 is due to the octahedral Fe only and corresponds to the $\nu_{2}$ band of $\mathrm{Fe}-\mathrm{O}$ of bulk magnetite $(\sim 370$ $\mathrm{cm}^{-1}$ ) shifted to a higher wavenumber. The intensity reduction of the band at $\sim 590 \mathrm{~cm}^{-1}$ from T90 to T33 is probably due to part of the $\mathrm{Fe}_{3} \mathrm{O}_{4}$ being reduced to elementary iron. ${ }^{61}$ The presence of $\mathrm{O}-\mathrm{H}$ stretching vibration at $\sim 3411 \mathrm{~cm}^{-1}$ and $\mathrm{O}-\mathrm{H}$ deformed vibration (bending modes) at $1630 \mathrm{~cm}^{-1}$ are attributed to the presence of coordinated $\mathrm{OH}$ groups or water molecules with the unsaturated surface Fe atoms. ${ }^{62} \mathrm{C}-\mathrm{O}$ stretching vibrations of $\mathrm{CO}_{3}^{=}$anion at $1400 \mathrm{~cm}^{-1}\left(\nu_{3}\right)$ in both the samples are due to atmospheric $\mathrm{CO}_{2} \cdot{ }^{63} \mathrm{~A}$ small absorption band at $2911 \mathrm{~cm}^{-1}$ is due to the $\nu_{\mathrm{s}}(-\mathrm{CH})$ vibrations from the residual

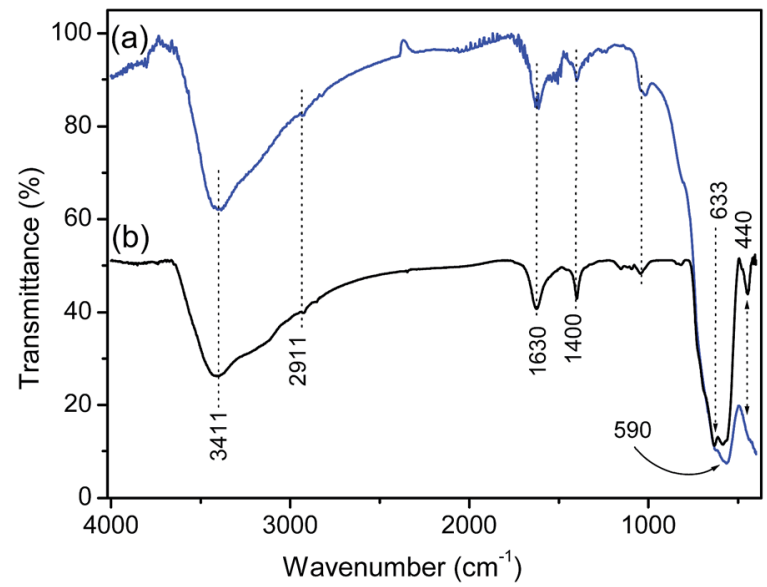

Fig. 5 FTIR spectra of the as-obtained (a) T33, and (b) T90 magnetite nanocrystals. solvents left after washing and drying steps. Raman spectra presented in Fig. S6† (see ESI) have also indicated the presence of predominantly magnetite with a small amount of maghemite $\left(\gamma-\mathrm{Fe}_{2} \mathrm{O}_{3}\right)$ as impurity in the black magnetic products (T75 and T90).

Fig. 6 shows the thermal analysis patterns (TG/DTA), nitrogen adsorption-desorption isotherms and the corresponding pore size distribution profiles of two representative magnetite specimens. The complete analyses of the isotherms are provided in Table 3. Both T33 and T90 showed hysteresis loops of type IV of Brunauer's classification, indicating the presence of mesopores in the powders.

The plot of $\mathrm{d} v / \mathrm{d} r$ versus pore size determined by the BarrettJoyner-Halenda (BJH) method shows that the pores (inset of Fig. 6A) are in the mesoporous range (5-10 nm) and is attributed to the interspaces of the constituent particles. It is obvious that the surface area of magnetite powders decreased with increase in size while increasing the precipitation temperature (Table 3$)$. The particle size $\left(D_{\mathrm{SA}}\right)$ measured by nitrogen adsorption is somewhat larger than the size estimated from TEM analysis (Fig. 1). This discrepancy can possibly be explained by agglomeration of smaller particles to form larger ones, thereby effectively reducing the collective surface area. The problem of agglomeration in dried NPs was particularly aggravated by the possible magnetic interactions and strong hydrogen bonding among them. The thermogram of magnetite (T90) shows a total weight loss of $\sim 3.8 \%$ in two stages on heating the powder sample to $1000{ }^{\circ} \mathrm{C}$. The first drop in the TG pattern at $\sim 100{ }^{\circ} \mathrm{C}$ is attributed to the physisorbed water remotion. The rate of loss increased till $\sim 500{ }^{\circ} \mathrm{C}$ and it could be attributed to the removal of multi-layers of water of hydration from the surface of the nanoparticles as well as dehydration of iron oxyhydroxide (FeOOH) formed by ambient moisture. A small weight gain of $\sim 0.4 \%$, observed in the temperature range $685-860{ }^{\circ} \mathrm{C}$ is due to oxidation of magnetite to $\gamma-\mathrm{Fe}_{2} \mathrm{O}_{3}$, though the experiment was conducted with continuous nitrogen purge. It is obvious that the thermal behavior of synthetic magnetite depends on the formation temperature, which affects the particle size. The finer magnetite crystals (e.g., 10-20 nm) rapidly undergo transition to maghemite at $\sim 150{ }^{\circ} \mathrm{C}$; whereas the larger magnetite particles are more thermally stable and do not start the transition to maghemite until about $315^{\circ} \mathrm{C}^{2}$

The mean hydrodynamic diameter ( $Z$-average) is based on the intensity of scattered photons from the colloidal particles in suspension. The $Z$-average size measured by dynamic light scattering of T60, is $36.2 \mathrm{~nm}$ with a polydispersity index (PDI) of 0.417 (Fig. S5 $\dagger$ ) though the $D_{\text {TEM }}$ for T60 is only $10.84 \mathrm{~nm}$. The PCS size is approximately 3 times larger than the physical size measured by TEM could be due to presence of one-shell hexagonal close-pack clusters of a total of maximum 13 particles with average hydrodynamic diameter of $\sim 3 \times D_{\text {TEM }}$ which is $32.5 \mathrm{~nm}$, close to $36.2 \mathrm{~nm}$. The zeta potential is the electrical potential measured at the shear plane, and represents the portion of the charge that can exert electrostatic attraction and repulsion forces on other particles. The T60 sample exhibit $\zeta$ values in the range +44.1 to $-43.9 \mathrm{mV}$ for the $\mathrm{NP}$ dispersion in the $\mathrm{pH}$ range 2.8 to 12 . The resulting aqueous dispersions were 

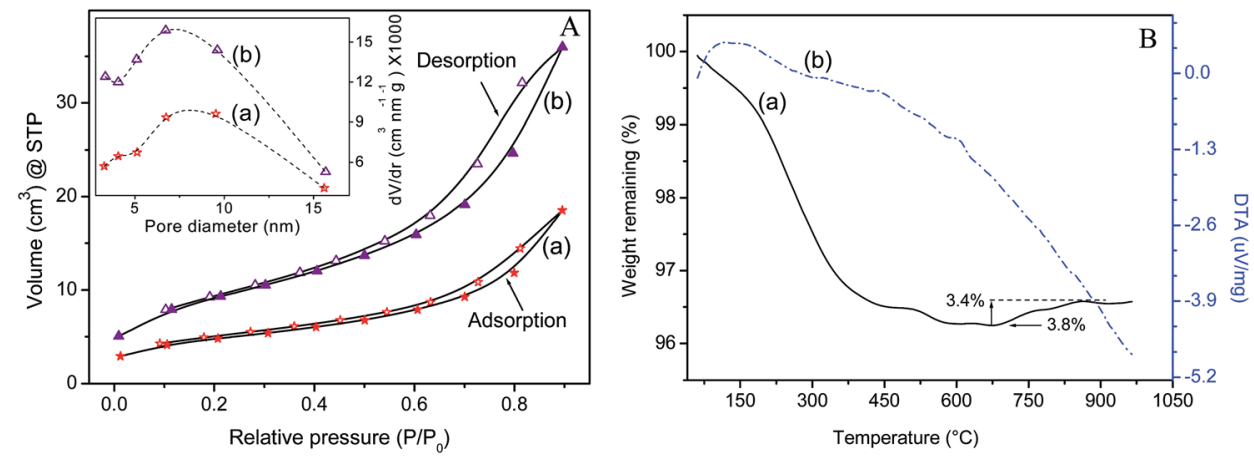

Fig. 6 (A) Volume $\mathrm{N}_{2}$ adsorbed versus relative pressure of $\mathrm{Fe}_{3} \mathrm{O}_{4}$ nanocrystals at $77 \mathrm{~K}$ for the powder samples (a) T90 and (b) T33. Inset shows the plot of pore size distribution derived from the BJH analysis. (B) Thermal analysis curves (a) TG and (b) differential thermal analysis representing the effects of thermal treatment on T90 in pure nitrogen atmosphere.

Table 3 Surface area, total pore volume, pore diameter and size of as synthesized magnetite in different conditions

\begin{tabular}{|c|c|c|c|c|c|c|}
\hline Sample & \multicolumn{4}{|c|}{ BJH pore distribution desorption } & $\begin{array}{l}\text { BET surface } \\
\text { area }\left(\mathrm{m}^{2} \mathrm{~g}^{-1}\right)\end{array}$ & Size, $D_{\mathrm{SA}}(\mathrm{nm})$ \\
\hline T90 & 51.252 & 0.106 & 9.531 & 0.1197 & 67.974 & 17.04 \\
\hline T75 & 75.917 & 0.145 & 6.782 & 0.1586 & 90.52 & 12.79 \\
\hline T60 & 89.601 & 0.174 & 5.141 & 0.1889 & 105.245 & 11.005 \\
\hline
\end{tabular}

stable in basic conditions, with the point of zero charge $\left(\mathrm{pH}_{\mathrm{PZC}}\right)$ at $\mathrm{pH} \sim 6.45$ which is close to already reported data. ${ }^{64}$ The corresponding surface reactions may be expressed as

$$
\mathrm{Fe}(\mathrm{II}, \mathrm{III}) \mathrm{OH}_{2}{ }^{+} \Longleftarrow \mathrm{H}^{\mathrm{H}^{+}} \mathrm{Fe}(\mathrm{II}, \mathrm{III}) \mathrm{OH} \rightleftharpoons \mathrm{Fe}(\mathrm{II}, \mathrm{III}) \mathrm{O}^{-}
$$

The resulting aqueous dispersions were stable in basic conditions. In acidic $\mathrm{pH}$, the dominating surface species is tentatively $\mathrm{Fe}(\mathrm{II}, \mathrm{III}) \mathrm{OH}_{2}{ }^{+}$, implying positive zeta potentials. With increasing $\mathrm{pH}$, the $\zeta$ decreases and $\mathrm{Fe}(\mathrm{II}, \mathrm{III}) \mathrm{OH}$ becomes dominating species around $\mathrm{pH}_{\mathrm{PZC}}$. At alkaline $\mathrm{pH}$, the surface species $\mathrm{Fe}(\mathrm{II}, \mathrm{III}) \mathrm{O}^{-}$is mainly responsible for the negative $\zeta .{ }^{65}$

\section{Conclusions}

Monodisperse magnetite nanoparticles with size in the range 6-13 $\mathrm{nm}$ were successfully synthesised by ammonia precipitation technique in the temperature range $33-90{ }^{\circ} \mathrm{C}$. The $\mathrm{X}-$ ray diffraction and the Rietveld refinement confirm the inverse spinel structure of cubic phase of magnetite as dominating phase. The activation energy for the growth of nanocrystallites during relatively higher temperature of precipitation was estimated to be $\sim 6.15 \mathrm{~kJ} \mathrm{~mol}^{-1}$ which is responsible for initiating the complex process of nucleation and growth by diffusion. $\mathrm{W}-\mathrm{H}$ technique indicated crystal dimensions within $D_{\text {TEM }}-2.5 \mathrm{~nm}$ as the shell of amorphous layer has not contributed to the X-ray diffraction. The lattice strains calculated were relatively high and in the range 5.5-
$8.9 \times 10^{-3}$ with very small variations for samples T33 to T90. The magnetic sizes ( $D_{\text {mag }}$ ) derived from the Langevin computations were smaller than the physical sizes from TEM analysis due to the supposedly presence of a magnetically "dead" layer of atoms over maghemite shell at the surface. Hence, precipitation method produced monodispersed magnetite nanocrystals with magnetite-maghemite coreshell structures suitable for biological applications.

\section{Acknowledgements}

The authors are grateful to the Director, Central Glass and Ceramic Research Institute, Kolkata for permission and extending facilities to carry out the above work. SJI and MJ acknowledge CSIR and UGC for their fellowships. Staff members of HR-TEM, XRD, and Central Instrumentation Facility are also acknowledged for their assistance in obtaining data. This work was supported by the 12 FYP CSIR Network project ESC-0103 and CSC-0135.

\section{Notes and references}

1 T. J. Daou, G. Pourroy, S. Begin-Colin, J. M. Greneche, C. Ulhaq-Bouillet, P. Legare, P. Bernhardt, C. Leuvrey and G. Rogez, Chem. Mater., 2006, 18, 4399.

2 H. T. Jeng and G. Y. Guo, Phys. Rev. B: Condens. Matter Mater. Phys., 2002, 65, 094429. 
3 H. M. Yang, C. W. Park, M. A. Woo, M. I. Kim, Y. M. Jo, H. G. Park and J. D. Kim, Biomacromolecules, 2010, 11, 2866. 4 Y. Piao, J. Kim, H. Bin Na, D. Kim, J. S. Baek, M. K. Ko, J. H. Lee, M. Shokouhimehr and T. Hyeon, Nat. Mater., 2008, 7, 242.

5 D. K. Kim, M. Mikhaylova, F. H. Wang, J. Kehr, B. Bjelke, Y. Zhang, T. Tsakalakos and M. Muhammed, Chem. Mater., 2003, 15, 4343.

6 M. O. Aviles, J. O. Mangual, A. D. Ebner and J. A. Ritter, Int. J. Pharm., 2008, 361, 202.

7 M. Chorny, I. Fishbein, B. B. Yellen, I. S. Alferiev, M. Bakay, S. Ganta, R. Adamo, M. Amiji, G. Friedman and R. J. Levy, Proc. Natl. Acad. Sci. U. S. A., 2010, 107, 8346.

8 S. H. Lee, Z. Y. Lu, S. V. Babu and E. Matijevic, J. Mater. Res., 2002, 17, 2744.

9 M. Uchida, M. L. Flenniken, M. Allen, D. A. Willits, B. E. Crowley, S. Brumfield, A. F. Willis, L. Jackiw, M. Jutila, M. J. Young and T. Douglas, J. Am. Chem. Soc., 2006, 128, 16626.

10 K. L. Lai, W. Jiang, J. Z. Tang, Y. Wu, B. He, G. Wang and Z. W. Gu, RSC Adv., 2012, 2, 13007.

11 K. Shimizu, A. Ito, J. K. Lee, T. Yoshida, K. Miwa, H. Ishiguro, Y. Numaguchi, T. Murohara, I. Kodama and H. Honda, Biotechnol. Bioeng., 2007, 96, 803.

12 L. M. Rossi, A. D. Quach and Z. Rosenzweig, Anal. Bioanal. Chem., 2004, 380, 606.

13 M. Shinkai, J. Biosci. Bioeng., 2002, 94, 606.

14 C. A. F. Vaz, J. Hoffman, A. B. Posadas and C. H. Ahn, Appl. Phys. Lett., 2009, 94, 022504.

15 H. F. Tian, T. L. Qu, L. B. Luo, J. J. Yang, S. M. Guo, H. Y. Zhang, Y. G. Zhao and J. Q. Li, Appl. Phys. Lett., 2008, 92, 063507.

16 D. Patel, A. Kell, B. Simard, J. X. Deng, B. Xiang, H. Y. Lin, M. Gruwel and G. H. Tian, Biomaterials, 2010, 31, 2866.

17 S. Ghosh, D. Carty, S. P. Clarke, S. A. Corr, R. Tekoriute, Y. K. Gun'ko and D. F. Brougham, Phys. Chem. Chem. Phys., 2010, 12, 14009.

18 S. Ghosh, D. Divya, K. C. Remani and T. S. Sreeremya, J. Nanopart. Res., 2010, 12, 1905.

19 U. Colombo, F. Gazzarrini and G. Lanzavecchia, Mater. Sci. Eng., 1967, 2, 125.

20 J. Tang, M. Myers, K. A. Bosnick and L. E. Brus, J. Phys. Chem. $B, 2003,107,7501$.

21 R. Frison, G. Cernuto, A. Cervellino, O. Zaharko, G. M. Colonna, A. Guagliardi and N. Masciocchi, Chem. Mater., 2013, 25, 4820.

22 S. C. Tjong and H. Chen, Mater. Sci. Eng., R, 2004, 45, 1.

23 G. A. Dorofeev, A. N. Streletskii, I. V. Povstugar, A. V. Protasov and E. P. Elsukov, Colloid J., 2012, 74, 675.

24 N. J. Tang, W. Zhong, H. Y. Jiang, X. L. Wu, W. Liu and Y. W. Du, J. Magn. Magn. Mater., 2004, 282, 92.

25 H. Wei, N. Insin, J. Lee, H. S. Han, J. M. Cordero, W. H. Liu and M. G. Bawendi, Nano Lett., 2012, 12, 22.

26 Y. F. Wang, Z. W. Zhu, F. Xu and X. L. Wei, J. Nanopart. Res., 2012, 14, 755.
27 J. Lee, Y. Lee, J. K. Youn, H. Bin Na, T. Yu, H. Kim, S. M. Lee, Y. M. Koo, J. H. Kwak, H. G. Park, H. N. Chang, M. Hwang, J. G. Park, J. Kim and T. Hyeon, Small, 2008, 4, 143.

28 J. Wan, W. Cai, X. Meng and E. Liu, Chem. Commun., 2007, 5004.

29 J. Pinkas, V. Reichlova, R. Zboril, Z. Moravec, P. Bezdicka and J. Matejkova, Ultrason. Sonochem., 2008, 15, 257.

30 S. Z. Wang, H. W. Xin and Y. T. Qian, Mater. Lett., 1997, 33, 113.

31 D. Vollath, D. V. Szabo, R. D. Taylor and J. O. Willis, J. Mater. Res., 1997, 12, 2175.

32 O. Masala and R. Seshadri, Annu. Rev. Mater. Res., 2004, 34, 41.

33 L. Signorini, L. Pasquini, L. Savini, R. Carboni, F. Boscherini, E. Bonetti, A. Giglia, M. Pedio, N. Mahne and S. Nannarone, Phys. Rev. B: Condens. Matter Mater. Phys., 2003, 68, 8.

34 L. F. Shen, P. E. Laibinis and T. A. Hatton, Langmuir, 1999, 15, 447 .

35 I. Nedkov, T. Merodiiska, L. Slavov, R. E. Vandenberghe, Y. Kusano and J. Takada, J. Magn. Magn. Mater., 2006, 300, 358.

36 B. L. Cushing, V. L. Kolesnichenko and C. J. O'Connor, Chem. Rev., 2004, 104, 3893.

37 H. M. Rietveld, Acta Crystallogr., 1966, 20, 508.

38 L. Lutterotti and P. Scardi, J. Appl. Crystallogr., 1990, 23, 246. 39 A. Krishnan, T. S. Sreeremya, E. Murray and S. Ghosh, J. Colloid Interface Sci., 2013, 389, 16.

40 C. P. Bean, J. Appl. Phys., 1955, 26, 1381.

41 C. P. Bean and I. S. Jacobs, J. Appl. Phys., 1956, 27, 1448.

42 C. P. Bean and J. D. Livingston, J. Appl. Phys., 1959, 30, S120.

43 V. Yathindranath, L. Rebbouh, D. F. Moore, D. W. Miller, J. van Lierop and T. Hegmann, Adv. Funct. Mater., 2011, 21, 1457.

44 Z. C. Xu, C. M. Shen, Y. A. Tian, X. Z. Shi and H. J. Gao, Nanoscale, 2010, 2, 1027.

45 Y. Tian, B. B. Yu, X. Li and K. Li, J. Mater. Chem., 2011, 21, 2476.

46 M. Respaud, J. Appl. Phys., 1999, 86, 556.

47 C. Liu and Z. J. Zhang, Chem. Mater., 2001, 13, 2092.

48 H. Shi, L. Tan, Q. Du, X. Chen, L. Li, T. Liu, C. Fu, H. Liu and X. Meng, Dalton Trans., 2014, 43, 12474.

49 S. W. Cao, Y. J. Zhu and J. Chang, New J. Chem., 2008, 32, 1526.

50 R. H. Kodama, J. Magn. Magn. Mater., 1999, 200, 359.

51 C. Pascal, J. L. Pascal, F. Favier, M. L. E. Moubtassim and C. Payen, Chem. Mater., 1999, 11, 141.

52 B. Y. Yu and S.-Y. Kwak, Dalton Trans., 2011, 40, 9989.

53 T. Ozkaya, M. S. Toprak, A. Baykal, H. Kavas, Y. Koseoglu and B. Aktas, J. Alloys Compd., 2009, 472, 18.

54 N. V. Lukashova, A. G. Savchenko, Y. D. Yagodkin, A. G. Muradova and E. V. Yurtov, Met. Sci. Heat Treat., 2013, 54, 550.

55 T. Narayanan, D. Sakthi Kumar, Y. Yoshida and M. Anantharaman, Bull. Mater. Sci., 2008, 31, 759.

56 C. Q. Sun, Nanoscale, 2010, 2, 1930.

57 T. S. Sreeremya, K. M. Thulasi, A. Krishnan and S. Ghosh, Ind. Eng. Chem. Res., 2012, 51, 318. 
58 C. Yang, J. J. Wu and Y. L. Hou, Chem. Commun., 2011, 47, 5130.

59 A. L. Andrade, D. M. Souza, M. C. Pereira, J. D. Fabris and R. Z. Domingues, Ceramica, 2009, 55, 420.

60 G. Marinescu, L. Patron, D. C. Culita, C. Neagoe, C. I. Lepadatu, I. Balint, L. Bessais and C. B. Cizmas, J. Nanopart. Res., 2006, 8, 1045.

61 Y. C. Dong, M. J. Hu, R. G. Ma, H. Cheng, S. L. Yang, Y. Y. Li and J. A. Zapien, CrystEngComm, 2013, 15, 1324.
62 A. Ebrahiminezhad, Y. Ghasemi, S. Rasoul-Amini, J. Barar and S. Davaran, Bull. Korean Chem. Soc., 2012, 33, 3957.

63 C. P. Chen, P. Gunawan and R. Xu, J. Mater. Chem., 2011, 21, 1218.

64 J. J. Carlson and S. K. Kawatra, Miner. Process. Extr. Metall. Rev., 2013, 34, 269.

65 Z. X. Sun, F. W. Su, W. Forsling and P. O. Samskog, J. Colloid Interface Sci., 1998, 197, 151. 